\title{
Editorial: Towards Improved Forecasting of Volcanic Eruptions
}

\section{F. Corentin Caudron", Lauriane Chardot', Társilo Girona ${ }^{3}$, ‥}

Yosuke Aoki ${ }^{4}$ and INico Fournier $^{5}$

- iUniv. Grenoble Alpes, Univ. Savoie Mont Blanc, CNRS, IRD, IFSTTAR, ISTerre, Gières, France

- ${ }^{2}$ Earth Observatory of Singapore, Nanyang Technological Institute, Singapore, Singapore

- 3 Jet Propulsion Laboratory, California Institute of Technology, Pasadena, CA, United States

- $\quad 4$ Earthquake Research Institute, University of Tokyo, Tokyo, Japan

- ${ }_{5}$ GNS Science, Lower Hutt, New Zealand

\section{Editorial on the Research Topic}

\section{Towards Improved Forecasting of Volcanic Eruptions}

Forecasting volcanic eruptions and their potential impacts are primary goals in Natural Hazards research. Active volcanoes are nowadays monitored by different ground and space-based instruments providing a wealth of seismic, geodetic, and chemical data for academic volcanologists and monitoring agencies. We have better insights into volcanic systems thanks to steady improvements in research tools and data processing techniques. The integration of these data into physics-based models allows us to constrain magma migration at depth and to derive the pressure evolution inside volcanic conduits and reservoirs, with the aim of ultimately forecasting volcanic eruptions.

Yet, it remains a challenge to answer the most crucial questions when the threat of an eruption looms over us: When will it occur? What will be its style and will it switch during the course of the eruption? How long will the eruption last? Most importantly: will we have enough time to alert and 
evacuate population? Addressing these questions is crucial to reduce the social and economic impact of volcanic eruptions, both at the local and global scales. For example, whilst the 2014 eruption at Ontake (Japan) impacted a relatively small surface area, dozens of hikers were killed due to their proximity to the eruptive vent; in contrast, the 2010 Eyjafjallajökull eruption (Iceland) did not cause any human loss yet paralyzed the European air space for weeks due to the resulting presence of ash in the atmosphere.

Several limitations arise when approaching the questions above. For instance, short-term eruption forecasts and models that relate changes in monitoring parameters to the probability, timing, and nature of future activity still bear a very high level of uncertainty. More reliable and useful quantitative forecasting requires substantial improvements both from a monitoring data accuracy and relevance standpoint, and their interpretation and modeling; only by developing optimized and integrated monitoring networks, along with statistical methods and models, the complexity of volcanic processes and system dynamics will be better captured and deciphered.

This Research Topic investigates these questions using multi-disciplinary approaches, challenges existing models and proposes some alternatives with the aim of improving the forecasting of volcanic eruptions and support decision making of local authorities. Below is the list of the 20 contributions to this volume.

Roman and Cashman challenge classic conduit formation models by reviewing the petrology and seismicity at six well-constrained case studies of arc volcanoes. They found that initial precursory seismicity is consistently several kilometers shallower than the magma reservoir and propose a new 3-phase model. In their model, precursory magma ascent could be detected well before the onset of seismic activity by continuous monitoring of the state of stress in the mid to shallow crust.

De Plaen et al. explore autocorrelations of ambient seismic noise at Mt. Etna volcano. They observed seismic velocity decreases accompanying 
paroxysmal eruptions, suggesting a significant pressurization within the plumbing system that caused some extensional strain and subsequent crack openings.

Lesage et al. investigate the existence of detectable precursors (surface displacements and seismic velocity variations) before the large dome collapse event of Volcán de Colima in July 2015. Their results show that no significant surface deformation or velocity change could be observed. Barrière et al. used seismic and infrasound signals generated by the Nyiragongo lava lake to quantify lava lake dynamic using a single sensor and SAR data to constrain lava lake levels. Drop of lake levels are reflected in changes in the seismo-acoustic signals, with the appearance of long period events probably resulting from deep lateral magma intrusions beneath Nyiragongo.

Salvage et al. analyse the seismo-volcanic events recorded prior to the 23 April 2017 at Poás in Costa Rica. Hindsight analysis revealed an acceleration within the dominant family of LF (low frequency) waveforms. However, no confidence could be placed in the forecast using the Failure Forecast Method (FFM), reiterating that not all accelerating trends are suitable for analysis using the FFM.

Brill et al. show a description of seismicity observed at Fuego volcano in Guatemala during January of 2012 and compute a 1-D velocity model to locate earthquakes. This work establishes a baseline of activity at this volcano.

Einarsson compiled seismic crises preceding 21 eruptions between 1973 and 2014. All eruptions were preceded by detectable precursor with lead time between $15 \mathrm{~min}$ and 13 days. These observations indicate that, under favorable conditions, seismic crises may be used for pre-eruption warnings. Pesicek et al. apply the $\beta$-statistic statistical tool to seismically monitored eruptions in Alaska of various styles to investigate seismic rate. Their results confirm that seismic rate increases are common prior to larger eruptions at long dormant, "closed-system" volcanoes, but uncommon preceding smaller eruptions at more frequently active, "open-system" volcanoes, with more mafic magmas. 
Londono and Galvis compared the volume of ash emission and seismic records of eruptions in Nevado del Ruiz volcano, Colombia, between 1985 and 2017. They found that the radiated seismic energy and reduced displacements can estimate the minimum ash loading, suggesting that seismic records may be used to monitor volcanic activity.

Campion et al. recorded $\mathrm{SO}_{2}$ emissions from the pit crater of Popocatépetl volcano between 2013 and 2016. The authors found that $>95 \%$ of the time the volcano is releasing gas passively at average rates of $45 \mathrm{~kg} / \mathrm{s}$ and with a dominant periodicity of $\sim 5$ min. Passive degassing was interspersed by small explosions with rapid return to pre-explosive levels and by strongest explosions which were preceded by rapid decreases of $\mathrm{SO}_{2}$ flux. Strong explosions are proposed to be triggered by the accumulation of gases beneath the lava dome of Popocatépetl.

Kilburn proposes a physical model relating faulting and elastic deformation as a function of loading rate, to explain precursory time series to eruptions. This elastic-brittle model is parent to the popular material Failure Forecast Method and may allow better forecasts of crustal failure.

Christophersen et al. explores the potential of Bayesian Networks (BNs) in modeling multiple data streams for eruption forecasting and volcanic hazard assessment. They show that $\mathrm{BN}$ modeling techniques require to accommodate continuous variables and link latent processes with observables and with eruptive patterns, and to model dynamic processes. Vasseur et al. demonstrate that mechanical heterogeneities control the accuracy of failure forecasts and that a minimum amount of data is required to reach convergence in the forecasts. They also propose a simple method to scale laboratory results to natural systems, using rupture length and frequency of seismic events.

Dzurisin explores the trigger mechanisms of the 1980-2006 and 20042008 Mount St. Helens eruptions. Both eruptions could have resulted from "second boiling" during crystallization of magma long-resident in an upper crustal reservoir, rather than from injection of fresh magma.

Peltier et al. shows changes in intensity, duration and time of appearance of long-term precursors, i.e., ground displacements and seismicity, for 43 
eruptions at Piton de la Fournaise (France). These findings ultimately improve the alerting chain and communication with decision-makers. Zhan et al. assimilate InSAR and GPS data using the Ensemble Kalman Filter. They provide a hindcast and derive the triggering mechanisms of the 2009 explosive eruption of Kerinci volcano (Indonesia) using a two-source analytical model.

Guldstrand et al. show how surface deformation induced by ascending eruptive feeders can be used to forecast the eruption location through a simple geometrical analysis. Their work builds on the results of 33 scaled laboratory experiments simulating magma intrusions in a brittle crust. Coombs et al. describe a multidisciplinary approach to forecast, rapidly detect, and characterize explosive events during the 2016-2017 eruption of Bogoslof volcano (Alaska). An effective strategy for hazard mitigation in remote areas is described.

Cameron et al. evaluate the timeliness and accuracy of eruption forecasts for 53 eruptions at 20 volcanoes in Alaska. They suggest that volcanospecific characteristics should be considered when designing monitoring programs and evaluating forecasting success.

Power and Cameron focus on the Alaska Volcano Observatory (AVO)'s response time, to identify seismic signals associated with large ashproducing volcanic explosions and initiate public warnings. While shorter response times were achieved during sequences of explosive events, longer response times are recorded for unexpected or surprise explosions. This Research Topic covered a wide range of methodologies and approaches which provide fundamental insights into (pre-)eruptive processes. Retrospective analyses of existing data and multi-disciplinary approaches may provide fundamental advances within the next few years. An obvious avenue of research concerns the tight link between observations and numerical modeling, ideally in near-real time using simple but efficient models. We also foresee an important improvement of forecasting thanks to satellite remote sensing data (e.g., Tropomi).

\section{Author Contributions}


All authors listed have made a substantial, direct and intellectual contribution to the work, and approved it for publication.

\section{Conflict of Interest}

The authors declare that the research was conducted in the absence of any commercial or financial relationships that could be construed as a potential conflict of interest. 